\title{
Effectiveness of a hydrogel dressing as an analgesic adjunct to first aid for the treatment of acute paediatric thermal burn injuries: study protocol for a randomised controlled trial
}

\author{
Maleea D. Holbert ${ }^{1,2^{*}}$ (D, Bronwyn R. Griffin ${ }^{1,2,7}$, Steven M. McPhail ${ }^{3,4}$, Robert S. Ware ${ }^{5}$, Kelly Foster ${ }^{6}$,
} Demi C. Bertoni ${ }^{2}$ and Roy M. Kimble ${ }^{1,2}$

\begin{abstract}
Background: Burns are a painful and traumatic experience, particularly in children. Reduced pain and anxiety positively influences re-epithelialisation rates in paediatric burn patients, however current literature fails to fully explain the effects of pain and anxiety and their links with wound healing. This study will determine if Burnaid ${ }^{\varpi}$ hydrogel dressing is an effective treatment for reducing pain in the acute period of a burn injury. It is hypothesised that a reduction in pain will then improve re-epithelialisation time in comparison to plastic wrap, which is standard practice at our institution - a metropolitan tertiary paediatric hospital located in Brisbane, Australia.

Methods/design: A randomised controlled trial will be conducted to assess the effectiveness of Burnaid ${ }^{\varpi}$ as an analgesic adjunct to cold running water first aid for the treatment of paediatric burns. Participants will include children aged between 0 and 16 years with an acute thermal burn injury (total burn surface area $<20 \%$ ) presenting to the Department of Emergency within $24 \mathrm{~h}$ of the burn occurring. Participants will be randomised into one of two groups: (1) Burnaid ${ }^{\varpi}$ hydrogel (intervention arm) or (2) plastic wrap (control arm). Participants will also be stratified into one of two groups based on factors that influence pain intensity: (1) high pain risk or (2) low pain risk. High pain risk factors include foot burns, hot coal/ash/fire pit burns, burn area greater than 5\%, and circumferential burns. The primary outcome is the intervention's effect on reducing acute pain. Secondary outcomes include days to re-epithelialisation, pulse rate, temperature, salivary cortisol and a-amylase, anxiety, and cost-effectiveness. Sample size calculations have shown that 36 participants will be recruited into each group.

Discussion: This study will provide comprehensive data on the analgesic properties of Burnaid ${ }^{\circledR}$ as an adjunct to first aid for the treatment of acute paediatric thermal burns. If the intervention is effective in reducing pain, Burnaid ${ }^{\circledR}$ will be integrated as standard practice within the hospital's Department of Emergency. This study replicates a real-world scenario in order to identify clinically significant analgesic and wound-healing effects.
\end{abstract}

Trial registration: Australian New Zealand Clinical Trials Registry, ACTRN12617001274369. Prospectively registered on 5 Sept 2017.

Keywords: Paediatric, Burns, First aid, Hydrogel, Burnaid®, Re-epithelialisation, Analgesia, Dressing, Randomised controlled trial, Pain

\footnotetext{
* Correspondence: m.holbert@uq.edu.au

${ }^{1}$ Centre for Children's Burns and Trauma Research, The University of

Queensland, Brisbane, Australia

${ }^{2}$ Pegg Leditschke Paediatric Burns Centre, Lady Cilento Children's Hospital,

South Brisbane, Australia

Full list of author information is available at the end of the article
}

(c) The Author(s). 2019 Open Access This article is distributed under the terms of the Creative Commons Attribution 4.0 International License (http://creativecommons.org/licenses/by/4.0/), which permits unrestricted use, distribution, and reproduction in any medium, provided you give appropriate credit to the original author(s) and the source, provide a link to the Creative Commons license, and indicate if changes were made. The Creative Commons Public Domain Dedication waiver (http://creativecommons.org/publicdomain/zero/1.0/) applies to the data made available in this article, unless otherwise stated. 


\section{Background}

Paediatric burn injuries pose a major epidemiological problem worldwide, with one-fourth of all burn injuries occurring in children under the age of 16 , the majority of whom are under 5 years of age [1]. Comprehensive management of burn wounds includes a challenging spectrum of acute, chronic, traumatic and surgical wounds with a wide range of anatomical locations and depths. Improving acute paediatric burn management is critical because untreated pain is thought to contribute to long-term sensory issues such as chronic pain, paraesthesia, dysesthesia and psychological conditions [2]. Moreover, optimal pain management may have significant implications with respect to re-epithelialisation rates in children with burn injuries [3].

Historically, burn injuries have been viewed as one of the most severe forms of trauma, and burn pain is considered one of the most severe forms of acute pain $[4,5]$. Burn treatment is also associated with significant pain and distress, and current standard treatments for such injuries have been insufficient in providing the analgesic needs for paediatric patients [6-9]. Paediatric burn patients undergo multiple painful and distressing procedures during their wound care and rehabilitation, which can cause severe long-term physiological and emotional effects [2]. If acute pain is not adequately managed, it can lead to altered long-term pain perception and maladaptive coping strategies, which can persist into adulthood [10-13]. Previous research also suggests that inadequate pain control can lead to the development of anticipatory anxiety for future medical procedures [9]. It is estimated that $10-20 \%$ of young patients who have sustained a burn injury go on to develop psychological disorders such as post-traumatic stress disorder [13, 14]. Therefore, optimising pain management during the acute phase of a paediatric burn is critical not only for the physical and cosmetic outcome of the injury but also for the child's well-being at the time and in the long term.

\section{First aid}

Correct first aid in the acute phase of a burn is essential for preventing further tissue damage, reducing pain in the acute phase and improving time to re-epithelialisation [15-20]. Immediate cooling via the use of cold running water (CRW) between $2{ }^{\circ} \mathrm{C}$ and $15^{\circ} \mathrm{C}$ for $20 \mathrm{~min}$ (applied within $3 \mathrm{~h}$ of the initial injury) is the recommended gold standard first aid for thermal burns, according to the Australian and New Zealand Burn Association [15, 17, 21, 22]. After adequate CRW the burn must be covered to protect the wound, reduce pain and prevent hypothermia [8]. The use of a hydrogel dressing that aids in cooling and hydration to the burn wound could be an ideal adjunct to current first aid practice. Advanced wound- dressing technologies require clinical data to support their use, and currently there is limited empirical evidence to support the use of hydrogel dressings as an analgesic adjunct to first aid for the treatment of acute burn injuries. Moreover, there are no studies investigating the use of these dressings in the paediatric burn population.

\section{Use of hydrogels on burns}

Current guidelines recommend burn wound covering after CRW first aid [8]. Acute burn wound coverings are required to be transparent and non-adherent and to have high application and removal ease. Plastic wrap fulfils these criteria and, excluding applications to the face, is a practical choice of dressing for acute burn injuries. In comparison to plastic wrap, Burnaid ${ }^{\circ}$ hydrogel dressings (Mundicare, Sydney, Australia) may provide additional pain relief via an evaporative cooling effect and may thereby assist in wound healing [23-25]; however, empirical evidence is still limited. The Burnaid ${ }^{\circ}$ hydrogel dressing comprises a 3-mm-thick, sterile, open foam polyester urethane pad that is impregnated with a propylene glycol gel which contains more than $90 \%$ purified water. Following the recognised standard of first aid treatment for acute burn injuries, Burnaid ${ }^{\circ}$ is recognised as an adjunct to first aid whose aim is to reduce pain. Moist interactive wound dressings provide pain relief via an evaporative cooling effect and create a moist environment which soothes exposed nerve endings in the skin [26]. Hydrogel dressings provide a concomitant cooling and wound-covering function and dissipate heat from the burn wound, and dressings come in a wide range of sizes which can be applied to all areas of the body [23, 27, 28].

The use of hydrogel dressings as an acute treatment for burn injuries has increased in the pre-hospital setting over the past decade. Allison showed that 39\% of emergency medical services (EMS) in the United Kingdom applied hydrogel dressings (e.g., Water-Jel, Water-Jel Technologies, Carlstadt, NJ, USA; Burnshield, Levtrade, Johannesburg, South Africa) during patient transport to hospital for burn injuries [29]. Walker and colleagues showed that $76 \%$ of UK fire and rescue services applied hydrogel dressings to cover burns, whereas $23 \%$ used plastic wrap as a burn wound dressing until EMS arrived [30]. Moreover, Cuttle and colleagues conducted an audit of first aid interventions for paediatric burn patients presenting to a tertiary burns centre located in Brisbane, Australia. This investigation found that Burnaid ${ }^{\circ}$ hydrogel dressings were applied by ambulance services in $12.9 \%$ of cases [31]. In addition, authors of a 2013 retrospective investigation reported that $5 \%$ of paediatric burns patients who presented to a tertiary burns centre in Sydney, Australia, had Burnaid ${ }^{\bullet}$ hydrogel dressings applied as a first aid dressing [32]. A large retrospective investigation 
into pre-hospital paediatric burn care was conducted in 2014 using data from electronic ambulance response forms for patients aged between 0 and 5 years who were attended by the Queensland Ambulance Service from 2008 to 2010 [18]. Data were collected from 117 paediatric burn incidents occurring within the Brisbane, Townsville and Cairns regions. Burnaid ${ }^{\circ}$ was the most commonly used dressing during patient transport and was applied to $55.6 \%$ of all paediatric burn patients. Brisbane paramedics applied Burnaid ${ }^{\circ}$ dressings in $44.3 \%$ of paediatric burn cases, whereas ambulance services in North and Far North Queensland applied Burnaid ${ }^{\circ}$ during patient transport in $72.7 \%$ and $60.9 \%$ of cases, respectively [18]. The difference in rates of use between regions further exemplifies the need for evidence-based guidelines for the use of hydrogel dressings in burn patients.

In a clinical investigation in which researchers evaluated the efficacy of hydrogel dressings in burn wound care, it was reported that hydrogel dressings conform well to the wound bed, are quick and easy to apply, and allow for wound inspection through the dressing [33]. In addition, it was observed that hydrogel dressings are effective for providing hydration to deep partial thickness burns and act to absorb exudate without becoming adherent to the wound bed. It was further reported that hydrogel sheet dressings are well tolerated by patients, who report no pain associated with dressing changes or between dressing changes [33]. The researchers in that study also commented on the paucity of evidence for the use of hydrogel dressings in burn care and their use in major burn wounds. This demonstrates the need for further investigation into the efficacy of hydrogel dressings for the acute treatment of burn injuries, so that evidence-based guidelines regarding their use and application can be developed. The potential that hydrogel dressings can reduce pain during the acute burn phase should not be underestimated in a clinical area where pain is most probably at its greatest. The primary aim of the present research is to assess the analgesic properties of Burnaid ${ }^{\circ}$ hydrogel dressings as an adjunct to current first aid in comparison to plastic wrap, which is currently used to cover burn injuries following CRW.

\section{Effect of pain and inflammatory mediators on wound healing}

The association between pain and wound healing is complex. In patients with significant tissue damage such as a burn, there is a prolonged and intense release of inflammatory mediators, and this can result in a state of hyperexcitability, which leads to an increased perception of pain [34]. It is evident that prolonged pain, such as that experienced by patients with significant burn injuries, can have maladaptive effects on wound healing time [35] and individual pain thresholds [2]. Prolonged release of inflammatory mediators from injured tissue acts to sensitise the inflamed area to pain via lowering the excitation threshold of nociceptors [34, 36]. This lowering of activation threshold (hyperalgesia) results in the perception of pain from typically non-painful stimuli and is often the case in burn patients who report increasing pain even where good progressive wound healing has occurred [36]. This further demonstrates the critical need to optimise pain management during the acute phase of a paediatric burn.

\section{Distraction, pain and wound healing}

Several studies describe the relationship between decreased pain and improved re-epithelialisation rates in paediatric burn patients $[3,6,37,38]$. A recent randomised controlled trial assessed the effectiveness of a procedural preparation and distraction device (Ditto $^{\mathrm{Tm}}$; Diversionary Therapy Technologies, Toowong, Australia) in reducing pain in paediatric burn patients during burn wound care [3]. This trial found that children who received the procedural preparation and distraction treatment reported significantly lower procedural pain scores (measured using the Wong-Baker Faces Pain Rating Scale) in comparison to patients who received standard care during their burn wound treatment $(p \leq 0.05)$. Parent and caregiver observational reports of procedural pain were also found to be lower in patients who received the preparation and distraction device $(p<0.001)$. Furthermore, children who received the Ditto ${ }^{\mathrm{TM}}$ device reepithelialised an average of 2.1 days faster than children in the standard care group (CI, $-4.38,0.10 ; p=0.061)$. Although not statistically significant, this result was clinically significant because the 2-day improvement rate in re-epithelialisation in patients who received the Ditto ${ }^{\text {тм }}$ treatment translated into a sizeable reduction in the need for scar monitoring: $26 \%$ of patients in the Ditto ${ }^{\mathrm{Ts}}$ treatment group re-epithelialised beyond 14 days, in contrast to $48 \%$ of participants in the standard practice group.

\section{Objectives}

The objective of the present study is to compare the effectiveness of plastic wrap (currently used to cover burns following CRW at Lady Cilento Children's Hospital ( $\mathrm{LCCH}$; Brisbane) and Burnaid ${ }^{\oplus}$ hydrogel dressing to reduce pain intensity in children who have sustained a burn injury prior to the application of a standard burns dressing.

\section{Hypotheses}

It is hypothesised that Burnaid ${ }^{\oplus}$ is more effective than plastic wrap at reducing pain intensity in the acute burn phase. It is also hypothesised that decreased pain experienced during the acute phase of the burn injury will speed up wound healing time and will 
result in a clinically significant reduction in days to full re-epithelialisation after application in children after a burn.

\section{Methods/design Design}

A single-centre, superiority, two-arm, parallel-group randomised controlled trial will be conducted to examine the effectiveness of Burnaid ${ }^{\circ}$ hydrogel dressing as an analgesic adjunct to first aid in comparison to plastic wrap. The present study includes methods that have been modelled on successful prior trials conducted by members of this investigative team at the same participating facility as the present trial $[39,40]$. The Consolidated Standards of Reporting Trials guidelines for randomised controlled trials will be followed, and the protocol adheres to the Standard Protocol Items: Recommendations for Interventional Trials guidelines (SPIRIT; see checklist in Additional file 1). Ethics approval for this project was obtained from LCCH and The University of Queensland human research ethics committees (HRECs). A flow diagram of the trial is provided in Fig. 1.

\section{Setting}

Recruitment will be conducted in the Department of Emergency (DEM) and Pegg Leditschke Children's Burns Centre Outpatient Department (OPD), located at LCCH, Brisbane. LCCH is categorised as a level 6 service, providing tertiary-level care for the state's most critically and seriously injured children.

\section{Inclusion criteria}

Children recruited into the study will be aged between 0 and 16 years and will have acquired an acute thermal burn injury that is $<20 \%$ of the total body surface area (TBSA). The child must present to the DEM or burns OPD within $24 \mathrm{~h}$ of sustaining the injury and must have no silver dressing application prior to this admission.

\section{Exclusion criteria}

Exclusion criteria for mechanism of injury are chemical, electrical, inhalation or friction burns; children who do not arrive within $24 \mathrm{~h}$ of sustaining their acute burn injury; children who do not receive appropriate first aid treatment; children who received prior treatment of a silver dressing or ointment; children who are non-Englishspeaking; children with cognitive impairments; children who are ventilated or require initial wound care in theatre for debridement or grafting; current involvement with the Department of Communities (child safety); children with known sensitivity to hydrogels or hypersensitivity; and children who have co-morbidities that would potentially impair would healing (e.g., diabetes) or that may exacerbate itch and pain.

\section{Recruitment}

Treating physicians/nursing staff of all children meeting the inclusion/exclusion criteria and presenting to the $\mathrm{LCCH}$, Brisbane, will determine eligibility for enrolment in the study. Medical staff, once establishing the child's eligibility, will then notify the parents/guardians and child of their eligibility to participant in the study. With parent/caregiver permission an investigator aligned with the study will discuss the trial with the parent/caregiver, seek informed consent from the parent/caregiver, and seek assent from the child (when appropriate). This trial will use a stratified sampling method taking into account factors which influence pain intensity in paediatric burn patients, based on anecdotal evidence and observations from paediatric burns surgeons and the unit's experience (unpublished hospital data audit). Once informed consent is obtained participants will be stratified into one of two groups: (1) high pain risk or (2) low pain risk. Participants presenting to LCCH DEM or burns OPD with one or more of the following criteria will be placed into the high pain risk group:

- Foot burns (unilateral or bilateral)

- Hot coal/ash/fire pit burns

- Circumferential burns

- $\mathrm{TBSA}>5 \%$

All other participants who meet the inclusion criteria for the trial but do not present with one or more of the four aforementioned pain risks will be placed in the low pain risk group. Following stratification, children will be randomised to one of the two treatment groups. Randomisation will be undertaken using a computerised random number sequence-generating programme, and treatment allocation will be masked by the use of sealed, opaque, identical, serially numbered envelopes prepared by an independent party. The researchers will not be blinded to which group the child is in, because they will be collecting set measures throughout the acute burn period. At any point throughout the study period the parent/guardian or child can withdraw from the study without any implications for the child's burn care, and all withdrawals and discontinuations will be recorded.

\section{Treatments}

\section{Intervention}

Burnaid ${ }^{\oplus}$ hydrogel dressing will serve as the treatment intervention in this trial. Paediatric burn patients randomised to the intervention arm will have the hydrogel dressing applied following $20 \mathrm{~min}$ of CRW, and the dressing will remain on the burn for a minimum of $20 \mathrm{~min}$. Burnaid $^{\circ}$ will be applied as per the manufacturer's instructions, followed by standard burn care, typically consisting 


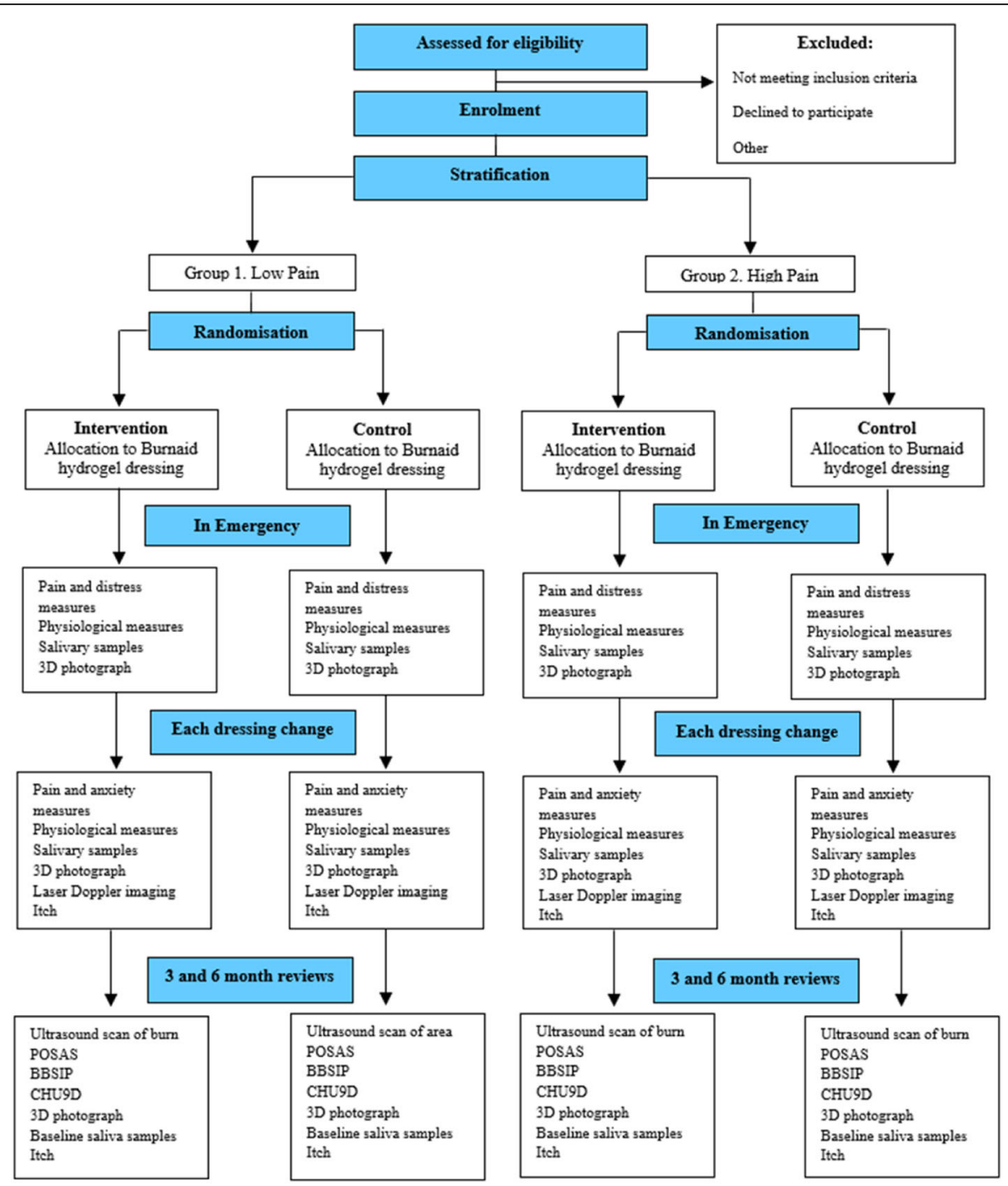

Fig. 1 Data collection flow diagram

of wound cleaning, debridement and silver dressing application.

\section{Control}

Plastic wrap is the current acute wound covering applied to thermal burn injuries at $\mathrm{LCCH}$, and it will serve as the control arm in this trial. Participants randomised to receive plastic wrap will have the dressing applied after first aid and will have the dressing remain on for a minimum of $20 \mathrm{~min}$, followed by standard burn care.

\section{Data collection}

Information regarding patient demographics and clinical details will be obtained from the parent/guardian, child (where age appropriate), and patient chart to record mechanism of injury, affected area, burn TBSA, what first aid was provided, form of transport, and time to presentation (see SPIRIT figure in Fig. 2). Apart from which type of dressing the child is receiving prior to the application of silver dressings, all participants will receive the standard medical care as would those not in the trial.

Following patient enrolment and randomisation, acute measures will be collected in LCCH DEM. Acute measures will include pain (measured using the Faces Pain Scale-Revised [FPS-R]; Face, Legs, Activity, Cry, Consolability scale [FLACC]; and visual analogue scale [VAS]), pulse rate, temperature, respiratory rate, and salivary cortisol and $\alpha$-amylase, which will be recorded by the primary investigator. Acute measures will be collected before and after the application of the randomised dressing and before and after silver dressing application from the child, parent/guardian, and nursing staff. In addition, peak FLACC scores during wound cleaning and debridement will be documented, as 


\begin{tabular}{|c|c|c|c|c|c|}
\hline \multirow[b]{3}{*}{ TIMEPOINT ** } & \multicolumn{5}{|c|}{ STUDY PERIOD } \\
\hline & \multirow{2}{*}{$\begin{array}{c}\begin{array}{c}\text { Enrolmen } \\
t\end{array} \\
-t_{1} \\
\end{array}$} & \multirow{2}{*}{$\begin{array}{c}\text { Allocation } \\
\mathbf{0}\end{array}$} & \multicolumn{3}{|c|}{ Post-allocation } \\
\hline & & & $t_{1}$ & $t_{2}$ & $t_{3}$ \\
\hline \multicolumn{6}{|l|}{ ENROLMENT: } \\
\hline Eligibility screen & $\mathrm{X}$ & & & & \\
\hline Informed consent & $\mathrm{X}$ & & & & \\
\hline Stratification & $\mathrm{X}$ & & & & \\
\hline Allocation to acute dressing group & & $\mathrm{X}$ & & & \\
\hline \multicolumn{6}{|l|}{ INTERVENTIONS: } \\
\hline \multirow{2}{*}{$\begin{array}{l}\text { Burnaid hydrogel dressing } \\
\text { Plastic wrap dressing }\end{array}$} & & $\mathrm{X}$ & & & \\
\hline & & $\mathrm{X}$ & & & \\
\hline \multicolumn{6}{|l|}{ ASSESSMENTS: } \\
\hline \multirow{2}{*}{$\begin{array}{l}\text { Demographic variables } \\
\text { Faces, Legs, Activity, Cry and Consolability } \\
\text { Pain Scale }\end{array}$} & $\mathrm{X}$ & $\mathrm{X}$ & & & \\
\hline & & $\mathrm{x}$ & $\mathrm{x}$ & $\mathrm{X}$ & $\mathrm{X}$ \\
\hline Visual Analogue Scale for Pain & & $\mathrm{X}$ & $\mathrm{X}$ & $\mathrm{X}$ & $\mathrm{X}$ \\
\hline \multirow[t]{2}{*}{ Faces Pain Scale-Revise } & & $\mathrm{X}$ & $\mathrm{X}$ & $\mathrm{X}$ & $\mathrm{X}$ \\
\hline & & $\mathrm{X}$ & $\mathrm{x}$ & & \\
\hline \multirow[t]{2}{*}{ Visual Analogue Scale for Anxiety } & & $\mathrm{X}$ & $\mathrm{x}$ & & \\
\hline & & $\mathrm{X}$ & $\mathrm{x}$ & $\mathrm{x}$ & \\
\hline Salivary measures of stress hormones & & & $\mathrm{X}$ & & \\
\hline \multirow{2}{*}{ Health Related Quality of Life } & & & & $\mathrm{X}$ & $\mathrm{X}$ \\
\hline & & & $\mathrm{X}$ & $\mathrm{X}$ & $\mathrm{X}$ \\
\hline \multirow[b]{2}{*}{ Need for surgical intervention post-injury } & & & & $\mathrm{X}$ & $\mathrm{X}$ \\
\hline & & & $\mathrm{x}$ & $\mathrm{X}$ & $\mathrm{X}$ \\
\hline
\end{tabular}

Fig. 2 SPIRIT figure. SPIRIT schedule of study recruitment, intervention and assessments. Allocation to the intervention group will occur in the Department of Emergency at Lady Cilento Children's Hospital upon patient admission following a thermal burn injury; $\mathrm{t} 1=3-5 \mathrm{days}$ after burn injury and each subsequent dressing change; $\mathrm{t} 2=3$ months after burn injury; $\mathrm{t} 3=6$ months after burn injury

will all analgesia and distraction techniques. All participants will have their burn wounds photographed using 3D imaging after wound debridement in the DEM to assess burn area. Patients (where appropriate) and parents/caregivers will be sent home with a diary to record pain scores and medications required prior to their first change of dressing (COD), which will typically occur 3 days after injury. Participants and parents/caregivers will be asked to bring this home diary with them to their first dressing change in the burns OPD.

Data collection in the burns OPD will continue through to the child's treating clinician classifying the burn wound as $95 \%$ re-epithelialised. Measures collected in the burns OPD at each dressing change appointment will include pain (measured using FPS-R, FLACC, and VAS), pulse rate, temperature, respiratory rate, and salivary cortisol and $\alpha$-amylase at each dressing change appointment. These measures will be collected before and after silver dressing removal and before and after the application of new silver dressings (see Fig. 4). Anxiety and itch will also be assessed at each COD appointment. At the first COD appointment, all participants will have their burn wound scanned using a non-invasive treatment referred to as laser Doppler imaging to measure burn depth and vascularity of the affected area. In addition, all participants will have a 3D image of their burn taken at each COD to assess the area of the wound and its rate of re-epithelialisation.

Following wound re-epithelialisation and discharge from the burns OPD, scar outcome will be assessed at 3 and 6 months post-burn for all participants. At each 3and 6-month follow-up, patients will have their burn wounds scanned using ultrasound to assess scar thickness; 3D imaging to assess scar area; DSM ColorMeter 
(Cortex Technology, Hadsund, Denmark) to examine scar colour and pigmentation; and Patient and Observer Scar Assessment Scale (POSAS), Brisbane Burn Scar Impact Profile (BBSIP) and Child Health Utility 9D Index (CHU9D) to measure health-related quality of life. Thy will also undergo itch assessment, and saliva samples will be collected to examine baseline cortisol and $\alpha$ amylase levels.

\section{Primary outcome measure}

The primary outcome of this trial is pain intensity after acute dressing application (see Measure 2 in Fig. 3). Observational reports from nursing staff using the FLACC scale will be used to measure this primary study outcome for all participants enrolled in this trial.

\section{Ancillary pain measures}

Pain intensity will be assessed before and after hydrogel dressing application and before and after silver dressing application, using self-report scales, parental/carer-specific, and nursing-specific pain scales (see Fig. 3). The set points of data collection will be recorded on a data collection form for patient and external observers to communicate pain intensity in a more accurate manner. The three physiological and psychological age-specific scales outlined below have been selected to allow assessment of pain and distress.

1. The FPS-R will be used to assess pain in children aged 4 years and older. The FPS-R is a self-rated, self-administered scale that uses images of facial expressions to assess pain intensity in children [41, 42]. The FPS-R has been shown to be superior to other faces scales [43] and has been validated in the literature as having a strong positive correlation $(r=0.93)$ with the visual analogue scale for pain (VAS-P) in children aged between 5 and 12 years [44]. Authors of a systematic review of self-report pain intensity measures for use in paediatric clinical trials reported that the FPS-R appears to be the most psychometrically sound pain assessment tool for children aged between 4 and 12 years [42]. Furthermore, a 2010 systematic review of the FPS-R for child self-reporting of pain intensity concluded that the FPS- $\mathrm{R}$ is recommended as a research tool because of the measure's utility and psychometric features [45].

2. The FLACC scale [46] will be used by nursing staff to assess pain and distress levels. The FLACC is an observational behavioural scale that has been validated for pain assessment in children aged between 2 months -7 years [46]. Observational pain scales such as the FLACC are widely used in paediatric clinical research [47]. A prospective observational investigation conducted in 2012 assessed the use of the FLACC scale to measure pain and distress in patients aged between 6 and 42 months $(N=125)$. This study found that FLACC scores can be high during non-painful procedures, as well as during the restraining phase of procedures [47]. These findings further support the notion that the FLACC assesses a composite of pain and distress in young children.

3. VAS-P for children aged 8 years and older and visual analogue scale observer (VASobs) for parent/guardian reports will be used. These scales have been chosen because they are age-specific and reliable and have been validated in recent literature. VASs have been validated and frequently

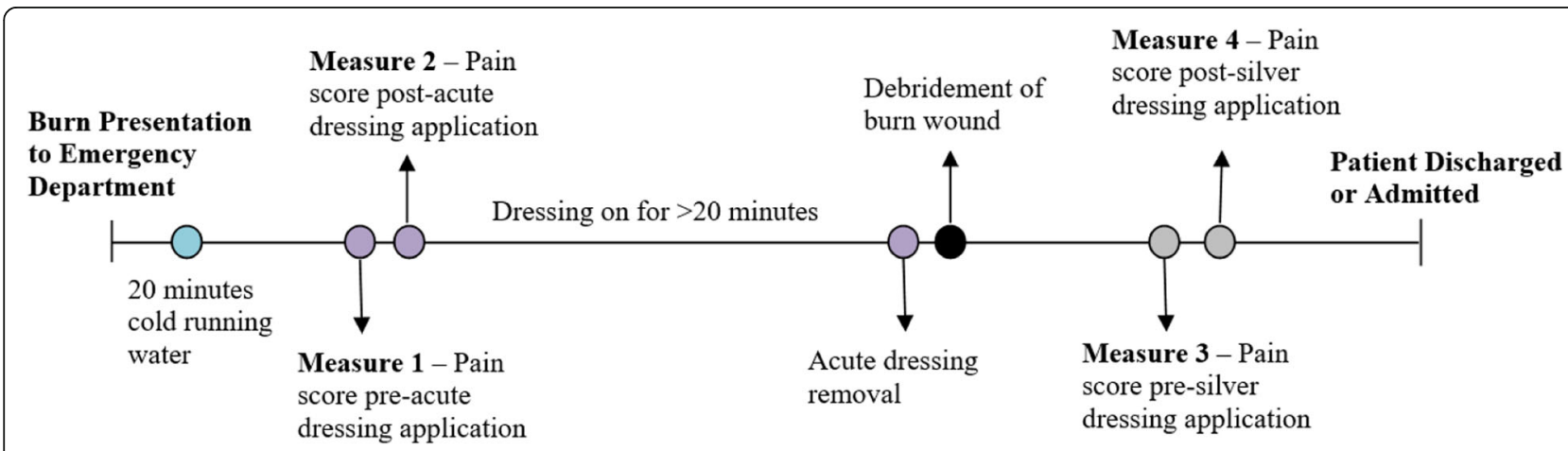

Fig. 3 Acute pain assessment in the Department of Emergency. Following cold running water first aid, pain will be assessed before and after the application of the randomised dressing, peak pain scores during wound cleaning and debridement, and before and after silver dressing application. Pain scores will be collected from the patient (where appropriate), observational pain scores will be collected from parents and caregivers, and Face, Legs, Activity, Cry, Consolability scale scores will be collected from nursing staff 
used to measure pain in adult populations and in paediatric populations as young as 5 years of age [48-50]. When quantifying pain intensity in preverbal and early-verbal children, who are unable to self-report, the VASobs repeatedly appears in the literature as a proxy for self-reports of pain [50-52]. Earlier studies have demonstrated that young burn patients can readily grasp the concept of quantifying their pain using anchored scales and even in moments of extreme discomfort are able to continue giving pain scores [53].

Physical measures of pain and distress will also be recorded at each of the measurement points depicted in Figs. 3 and 4. These measures include pulse rate, respiratory rate and temperature because increases in these physiological measures have been shown to be indicative of pain and distress.

\section{Secondary outcome measures \\ Days to re-epithelialisation}

The number of days from the date of the initial burn until 95\% wound re-epithelialisation occurs, the surface area of the affected area, and the percentage of wound re-epithelialisation will be calculated using three methods: (1) clinical judgment of the treating consultant, (2) 3D photography (3D LifeViz ${ }^{\text {тм }}$ System; QuantifiCare, Valbonne, France) and analysis using specialist computer software, and (3) blinded review of the 3D photographs by a panel of burn specialists. The 3D LifeViz ${ }^{\text {TM }}$ System will be used to photograph all burns upon first presentation to the hospital following wound debridement and at each subsequent burns OPD visit for dressing changes and wound care. This will be performed for all participants until burn wounds are deemed to be $95 \%$ re-epithelialised or are grafted. Research has found 3D photography to be a valid and reliable measure of acute burn wound surface area in an Australian paediatric population $[54,55]$. In order to ensure accurate measurement calibration in the associated DermaPix ${ }^{\mathrm{TM}}$ software package (QuantifiCare), a ruler will also be included in all 3D photographs. The 3D images will be analysed by the primary investigator using the DermaPix software programme to determine the surface area of the burn and the area of re-epithelialisation of the wound at each COD. A panel of burn specialists, which will be composed of consultants and nursing staff, will perform a blinded review of the 3D images and will assess the level of re-epithelialisation and appearance of each participant's burn, mapping out the edges of the wound and all unhealed areas. We will further examine concordance between the burn specialist's clinical assessment and the DermaPix mapping programme regarding re-epithelialisation.

\section{Staff and caregiver perspectives on dressings}

Perception of ease of dressing application, removal, flexibility, and conformity will be rated by medical and nursing staff using a self-reported 0-10 Numeric Rating Scale (NRS) for both the Burnaid ${ }^{\circledR}$ hydrogel dressing and plastic wrap groups. Clinical staff will also be surveyed again using the same NRS after recruitment has ceased in order to examine any changes in their perception of either acute wound covering. In addition, parent/caregiver and patient (when appropriate) perception of comfort, ease of movement, and ease of dressing application/ removal will be measured using an NRS. It is noted that ease of dressing measurements in the DEM will be confounded owing to lack of blinding and as a result of the variable nature, size, and anatomical location of the areas to be dressed.

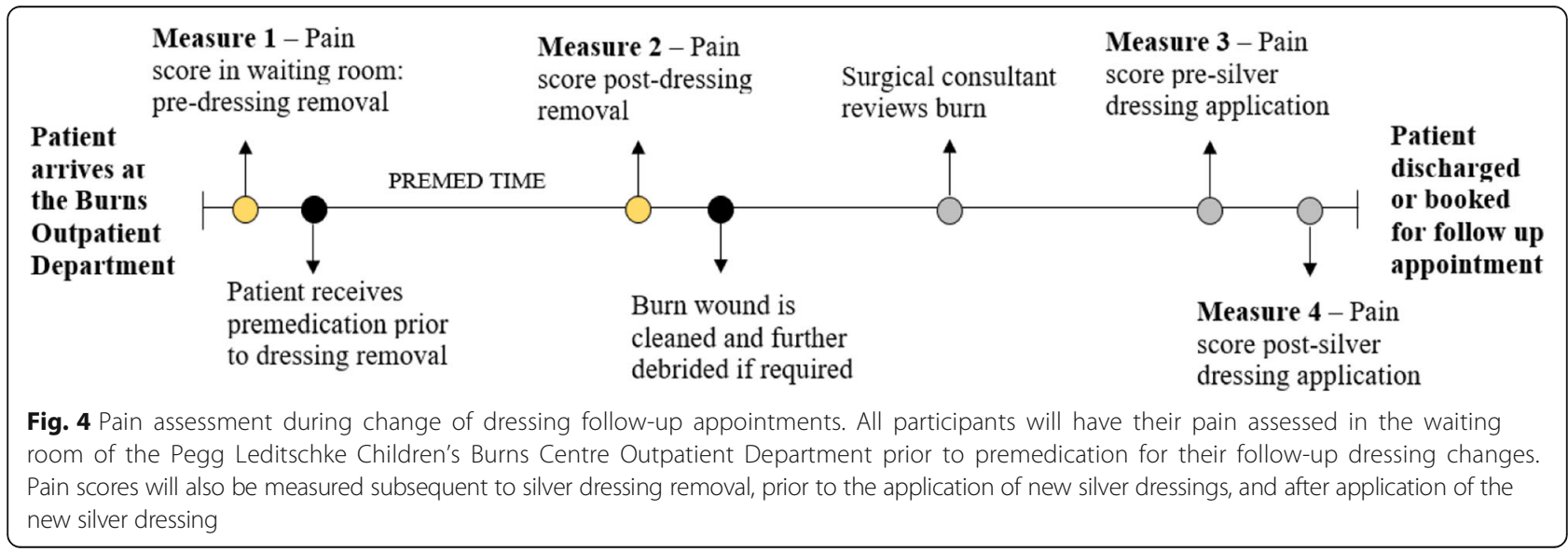




\section{Scar/skin condition at 3 and 6 months}

At 3 and 6 months after the date of injury, a face-to-face follow-up will be performed with all participants (intervention and standard care groups) to assess the skin and scar characteristics of the affected area. This assessment will involve (1) 3D camera imaging (3D $\mathrm{LifeViz}^{\mathrm{Tm}}$ System), which will be used to determine scar area and volume above the skin; (2) ultrasound scanning (BT12 Venue 40 MSK; GE Healthcare, Chicago, IL, USA), which will be used to measure the height of the scar; and (3) the POSAS measurement tool, which will be used to assess scar severity. The POSAS will be completed with the patient (if aged 6 years or older) and the parent/caregiver, and it will document observer scale items of scar thickness, vascularity, pliability, pigmentation and relief in addition to patient scale items of itch, pain, colour, stiffness, thickness and irregularity. In addition, DSM II Skin ColorMeter analysis will be performed to assess the lightness, redness or erythema, and pigmentation of the scar. The DSM II ColorMeter is an objective tool that uses tri-stimulus reflectance colourimetry and narrow-band photometry to assess scar characteristics, and it has been demonstrated to have inter-observer reliability intra-class correlation coefficients of 0.94 for lightness, 0.94 for erythema and 0.83 for pigmentation in scar assessment [56].

\section{Pain frequency}

The BBSIP will be used to assess the intensity and frequency of sensations, including pain, tightness and discomfort, in addition to health-related quality of life attributes which are specific to individuals with burn scars. The BBSIP provides unique insight into health-related quality of life in children with burn scarring, in addition to the concerns of parents/caregivers of children with burn scaring. It has been shown to have preliminary content validity, and further research is currently being conducted in children, adults and parents/caregivers of children with burn scars [57].

\section{Itch}

The Toronto Paediatric Itch Scale (TPIS) will be used to assess pruritus in patients younger than 5 years of age. The TPIS is a behaviour-anchored post-burn itch scale which has been demonstrated in the literature to be a valid and reliable measurement tool with a moderate inter-observer agreement (Cohen's kappa of 0.52, $p<0.001$ ) [58]. Parents/caregivers of patients younger than 5 years old will complete the TPIS, which rates itch behaviours on a scale of 0 (indicating absence of itch) to 3 (which represents severe itch with significant disruption). In addition to the aforementioned factors, this scale was selected because it has been shown to be user-friendly, intuitive and reliable for use in infants and children as young as 5 years old or less [58]. Participants 5 years of age and older will self-report their itch intensity using a $0-10$ NRS. In addition, all caregivers will complete the itch component of the BBSIP. Itch will be assessed at the first COD and each follow-up until the wound is deemed re-epithelised, and it will also be assessed at 3- and 6-month reviews.

\section{Health-related quality of life}

The CHU9D is a generic preference-based instrument designed for use in children and adolescents to assess how their health affects their life. The CHU9D will be used to measure health-related quality of life in a way that is suitable for use in cost-utility analyses. Dimensions of the CHU9D include worry, sadness, pain, fatigue, annoyance, schoolwork/homework, sleep, daily routine and ability to partake in activities. Preference weights applied to these nine attributes enable the calculation of a multi-attribute utility score where 0 represents death and 1 represents full health [59]. The CHU9D has been developed and validated for young people aged between 7 and 17 years, and it has been shown to have good construct validity within an Australian adolescent population [60]. At each 3- and 6-month post-burn follow-up, children will self-complete the CHU9D as appropriate, and a parent/ caregiver proxy-report will be completed for all participants (children of all ages).

\section{Stress and anxiety}

Stress is a secondary outcome and will be quantified via salivary cortisol and $\alpha$-amylase. Salivary cortisol is often used as a biomarker to assess stress response and is considered to be a reliable index of hypothalamic-pituitary-adrenal axis activity [61]. The measurement of cortisol levels in saliva is the preferred method of assessment for researchers, particularly in a paediatric population; salivary analysis is non-invasive and painfree in comparison to blood sampling and therefore does not induce additional stress and trauma (and potentially higher cortisol) as occurs with serum sampling [62]. Moreover, there is a strong correlation between cortisol levels in saliva and unbound cortisol in plasma [63]. Salivary $\alpha$-amylase is also said to be a reliable, valid and sensitive biomarker for stress-related changes in the autonomic nervous system and is a proxy for noradrenaline, which is a sign of sympathetic adrenomedullary system activation [64].

Participants will place a SalivaBio Oral Swab ${ }^{\text {tw }}$ (Salimetrics Europe Ltd., Newmarket, UK) under their tongue for $2 \mathrm{~min}$ for saliva collection. Salivary stress biomarkers will be collected at the following time points: 
1. At first presentation to the DEM or burns OPD subsequent to the application of either Burnaid ${ }^{\circ}$ hydrogel dressings or plastic wrap

2. Immediately after the removal of the Burnaid ${ }^{\circ}$ or plastic wrap dressing

3. Before application of silver dressings

4. Immediately after the application of silver dressings

5. Immediately before premedication prior to the patient's first COD

6. Immediately before and after silver dressing removal

7. After application of the new silver dressings

8. Three months after burn injury to obtain a baseline

Parents/caregivers and patients (where appropriate) will also complete a saliva collection survey which documents variables pertinent to salivary analysis. Such variables include all medications given to the patient, time the patient last woke up, time the patient last brushed their teeth, all food/drink/gum consumed by the patient during the previous hour, time the participant last consumed caffeine, pertinent smoking/tobacco history, and sample collection time. The date, time and volume of saliva collection will be recorded in the laboratory, and samples will be refrigerated at $4{ }^{\circ} \mathrm{C}$ and processed within 3 days of collection. Samples will be spun in a centrifuge at $1400 \times g$ at room temperature for $15 \mathrm{~min}$, and the saliva will be frozen at $-80^{\circ} \mathrm{C}$ until analysis. Salivary cortisol and $\alpha$-amylase will be quantified using enzyme-linked immunosorbent assay kits (Stratech Scientific, Ely, UK), with saliva samples analysed in triplicate to ensure accurate results.

\section{Healthcare resources and costs}

Healthcare resource use will be collected from the perspective of a health service provider and costed at market rates using methods that have previously been employed in trials by members of this research team at this participating centre $[65,66]$. This includes recording trial intervention-related resource use (e.g., the types and amounts of acute wound dressings used) as well as other burn-related healthcare resources that may be important to a health service when deciding which of the interventions to implement in clinical services providing care for children with acute burns. Clinician labour time will be recorded for each participant and costed at state award rates for each respective discipline.

\section{Blinding}

Owing to the nature of the trial, blinding will not be possible, because treating clinicians, nursing staff, patients and their families will be aware of the intervention. The primary investigator will be present when the acute wound dressings are being applied and removed to obtain pain scores and additional measures from the participant, caregiver and nursing staff. The acute wound covering applied to each participant cannot be masked within this environment, and therefore investigator blinding will not be possible. In order to incorporate an element of blinding in the trial, an expert panel of burn wound specialist will conduct a blinded review of 3D photographs for all participants to assess burn wound depth and re-epithelialisation at each dressing change until complete wound healing. Wherever possible during data collection analysis, treatment groups will be de-identified.

\section{Adverse effects}

The proposed intervention is considered to be part of standard pre-hospital care and is currently used by Queensland Ambulance Services, despite Burnaid ${ }^{\circ}$ products not being used at the LCCH DEM and burns OPD. Therefore, minimal adverse events are expected. Known potential adverse events (such as hypothermia, infection, haematoma, excessive exudates, unpleasant sensations, allergic reactions, chondritis, and disruption of the skin on dressing removal) have a standardised management protocol at LCCH DEM and burns OPD. In addition, children with known adverse reactions to hydrogels will be excluded from the trial. Adverse effects of the hydrogel dressing will be monitored by reviewing patient medical records and via self-report data from parents/ caregivers, participants (where appropriate), and treating clinicians. All adverse events will be reported to the clinical health service and the overseeing HREC. If a consultant believes that Burnaid $^{\circ}$ hydrogel dressings were not appropriate for a patient's treatment, discontinuation or alteration of treatment will be at their discretion, and data collection for such participants will cease from that time point.

\section{Data monitoring}

Regular team meetings will be held to monitor the progress of the study and set timelines and will allow for any issues to be discussed and resolved. All data will remain secure and de-identified, which the primary investigator will ensure in addition to data cleansing and circulation of results/outcomes.

\section{Data storage}

Collected electronic data will remain secure through password protection on University of Queensland servers. Filing cabinets containing patient data will remain locked and secured within the swipe card-accessed Centre for Children's Health Research. All data collected for the purpose of this investigation will be accessible only by the primary investigator and approved names on the ethics application form. Data collected and used for the trial will 
be de-identified, cleaned and checked. Any missing data will be coded as missing, unknown or not applicable before being locked for analysis. Data will remain secure for 15 years as per the requirements of The University of Queensland HREC.

\section{Sample size}

The sample size estimate was calculated on the basis of the primary outcome of pain intensity after dressing application. Previous researchers assessing paediatric burn pain reported that pain scores within each subject group were normally distributed with an SD of 2.4 [37]. To detect a significant between-group difference of 1.8 in pain scores after dressing application, 29 experimental participants and 29 control participants will need to be recruited in order to reject the null hypothesis that the population means of the experimental and control groups are equal with probability (power) of 0.8 . The type I error probability associated with the test of this null hypothesis is 0.05 . With up to a potential $20 \%$ loss to follow-up, the calculated target sample size is equal to 72 participants. LCCH treats approximately $25-34$ children per month in the DEM for acute burn injury. On the basis of these estimates and a historical 70\% success rate for trial recruitment within $\mathrm{LCCH}$, it is predicted that around 17 patients will be recruited per month. Therefore, it is estimated that data collection should be completed within 12 months from recruitment commencement.

\section{Data analysis}

The data set will be analysed using IBM SPSS Statistics software (version 22; IBM, Armonk, NY, USA) and Stata software (StataCorp, College Station, TX, USA). Descriptive statistics will be calculated for all outcomes. Univariate parametric analyses (e.g., $t$ test, chi-square test) or alternative non-parametric equivalent tests (e.g., MannWhitney $U$ test) will be used to assess differences between groups at baseline where appropriate. Betweengroup differences in outcomes will be analysed using regression models. When analyses include multiple measures on the same participant, mixed effects methods will be used to account for probable non-independence in observations. When outcomes are on an interval scale, linear models will be used. When outcomes are binary, logistic models will be used, and when outcomes are recorded as count data, Poisson models will be used. Sensitivity analyses will be undertaken using multiple imputation methods, where it can be assumed data are missing at random. Analysis of differences between groups, and also differences within groups over time, will be undertaken. Analyses will be done on an intentionto-treat basis. Significance will be set at $p<0.05$.

\section{Discussion}

Pain and distress following first aid remains a major challenge when treating acute paediatric burn injuries, and literature shows that increased pain and stress can have maladaptive effects on wound healing $[35,37,67]$ and patient outcomes [68]. Although Burnaid ${ }^{\circ}$ hydrogel dressings are frequently being used by Queensland Ambulance Services during patient transport to hospital for burn injuries [18], a limited number of high-level studies have been conducted examining the clinical utility of these dressings, particularly in relation to analgesic properties and wound-healing effects. The widespread use of hydrogel burn dressings (such as Burnaid ${ }^{\circ}$, BurnShield ${ }^{\circ}$, and Water-Jel ${ }^{\circ}$ ) is alarming owing to the lack of research supporting their use as a first aid dressing [69]. This randomised controlled trial will provide high-level evidence to determine if Burnaid ${ }^{\oplus}$ hydrogel dressing provides superior pain relief in comparison to plastic wrap as an acute burn wound covering applied after first aid in paediatric patients with thermal burn injuries. Establishing which dressing is more effective in reducing acute pain is critical to clinical care and also to facilitate further evidence-based guidelines in this field.

Burns are a painful and traumatic experience, particularly in children. Considering the adverse psychological and physiological effects secondary to pain, optimal pain management should be viewed as an essential and requisite component in acute paediatric burn treatment [9]. Reduced pain and anxiety positively influences re-epithelialisation rates [3], and time to re-epithelialisation is a good predictor of scar outcome in patients with burn injuries [70]. This further validates attempts to speed up re-epithelialisation rates. Even if hydrogel products are more expensive than current standard acute wound coverings, the cost of treating adverse scarring is substantial in resources and time, as well as in cosmetic outcome for the patient. Burnaid ${ }^{\circ}$ hydrogel dressings have been shown to be effective evaporative cooling agents, which is why these dressings are hypothesised to have an analgesic effect during acute burn care [69].

\section{Trial status}

Recruitment will commence at the beginning of September 2017, and it is expected that recruitment will take approximately 12 months to complete, with final data collection occurring in January 2019.

\section{Additional file}

\section{Additional file 1: SPIRIT checklist. (DOC $124 \mathrm{~kb}$ )}

Abbreviations

BBSIP: Brisbane Burn Scar Impact Profile; CHU9D: Child Health Utility 9D Index; COD: Change of dressing; CRW: Cold running water; 
DEM: Department of Emergency; EMS: Emergency medical services; FLACC: Face, Legs, Activity, Cry, Consolability scale; FPS-R: Faces Pain Scale-Revised; HREC: Human Research Ethics Committee; LCCH: Lady Cilento Children's Hospital; NRS: Numeric Rating Scale; OPD: Outpatient department; POSAS: Patient and Observer Scar Assessment Scale; SPIRIT: Standard Protocol Items: Recommendations for Interventional Trials; TBSA: Total body surface area; TPIS: Toronto Paediatric Itch Scale; VAS: Visual analogue scale; VASobs: Visual analogue scale observer; VAS P: Visual analogue scale for pain

\section{Acknowledgements}

The authors acknowledge all the children, families and health professionals who will participate in this trial, as well as all the staff at the Pegg Leditschke Children's Burns Centre and Department of Emergency at the Lady Cilento Children's Hospital, Brisbane, Australia, for their support and assistance throughout data collection. This study is funded by an Australian Government Research Training Scholarship and a grant given to The University of Queensland by Mundipharma. SMM is supported by a National Health and Medical Research Council of Australia fellowship.

\section{Funding}

This study is funded by an Australian Government Research Training Scholarship to the author $\mathrm{MH}$ and a research grant provided to The University of Queensland by Mundipharma.

\section{Availability of data and materials}

Not applicable for this protocol manuscript.

\section{Authors' contributions}

BRG and RMK made substantial contributions to the design of this trial. RSW $B R G$, SMM and DCB made substantial contributions to the analysis plan for this investigation. $\mathrm{MDH}$ wrote the draft manuscript with substantial input from BRG and DCB. BRG, RMK, RSW, KF, SMM and DCB provided critical review of the manuscript. All authors read and approved the final manuscript.

\section{Ethics approval and consent to participate}

This trial has being granted ethical approval by The University of Queensland Human Research Ethics Committee (HREC/16/QRCH/322), LCCH (Brisbane) Children's Health Service District Ethics Committee and the Children's Health Queensland Human Research Ethics Committee. The study methodology was documented in a protocol and registered with the Australian New Zealand Clinical Trials Registry (ACTRN12617001274369) on 5 September 2017 prior to starting recruitment. All participants will be given verbal and written information about the study and will provide signed consent to participate in the research. The information collected about participants is individually identifiable by members of the research team only. Each participant will be allocated a unique numeric code (ID Number) such that all stored electronic data (e.g., databases, data files) will contain identifiable data until the completion of the study. All confidential information is stored in locked filing cabinets, and only deidentified data will be presented or published.

\section{Consent for publication}

Written informed consent will be obtained from the parent/guardian or participant where applicable for accompanying images in published reports of this study. The consent form is held by the authors and is available for review by the Editor-in-Chief of this journal. It is standard for all clinical trials conducted within the Centre for Children's Burns and Trauma Research to publish a protocol in Trials, as well as all results of the trial being published in journals and presented at conferences both nationally and internationally.

\section{Competing interests}

The authors declare that they have no competing interests.

\section{Publisher's Note}

Springer Nature remains neutral with regard to jurisdictional claims in published maps and institutional affiliations.

\section{Author details}

${ }^{1}$ Centre for Children's Burns and Trauma Research, The University of Queensland, Brisbane, Australia. ${ }^{2}$ Pegg Leditschke Paediatric Burns Centre, Lady Cilento Children's Hospital, South Brisbane, Australia. ${ }^{3}$ Centre for Functioning and Health Research, Metro South Health, Buranda, Australia. ${ }^{4}$ School of Public Health \& Social Work and Institute of Health and Biomedical Innovation, Queensland University of Technology, Brisbane, Australia. ${ }^{5}$ Menzies Health Institute Queensland, Griffith University, Brisbane, Australia. ${ }^{6}$ Paediatric Emergency Research Unit, Children's Health Queensland, South Brisbane, Australia. ${ }^{7}$ School of Nursing, Queensland University of Technology, Brisbane, Australia.

Received: 23 October 2017 Accepted: 16 November 2018 Published online: 06 January 2019

References

1. Bayat A, Ramaiah R, Bhananker SM. Analgesia and sedation for children undergoing burn wound care. Expert Rev Neurother. 2010;10(11):1747-59.

2. Summer GJ, Puntillo KA, Miaskowski C, Green PG, Levine JD. Burn injury pain: the continuing challenge. J Pain. 2007;8(7):533-48.

3. Brown NJ, Kimble RM, Rodger S, Ware RS, Cuttle L. Play and heal: randomized controlled trial of Ditto ${ }^{\mathrm{TM}}$ intervention efficacy on improving re-epithelialization in pediatric burns. Burns. 2013;40(2):204-13.

4. Chapman CR, Bonica JJ. Acute pain. Kalamazoo: Upjohn; 1983.

5. Pal SK, Cortiella J, Herndon D. Adjunctive methods of pain control in burns. Burns. 1997:23(5):404-12.

6. Miller K, Rodger S, Bucolo S, Greer R, Kimble RM. Multi-modal distraction: using technology to combat pain in young children with burn injuries. Burns. 2010;36(5):647-58

7. Hoffman HG, Patterson DR, Magula J, Carrougher GJ, Zeltzer K, Dagadakis S, et al. Water-friendly virtual reality pain control during wound care. J Clin Psychol. 2004;60(2):189-95

8. Baartmans MGA, de Jong AEE, van Baar ME, Beerthuizen GIJM, van Loey NEE, Tibboel D, et al. Early management in children with burns: cooling, wound care and pain management. Burns. 2016;42(4):777-82.

9. Gandhi M, Thomson C, Lord D, Enoch S. Management of pain in children with burns. Int J Pediatr. 2010;2010:825657.

10. Howard RF. Current status of pain management in children. JAMA. 2003; 290(18):2464-9.

11. Young KD. Pediatric procedural pain. Ann Emerg Med. 2005;45(2):160-71.

12. Thurber CA, Martin-Herz SP, Patterson DR. Psychological principles of burn wound pain in children. I: theoretical framework. J Burn Care Rehabil. 2000; 21(4):376-87 discussion 375.

13. Saxe G, Stoddard F, Hall E, Chawla N, Lopez C, Sheridan R, et al. Pathways to PTSD, part I: children with burns. Am J Psychiatry. 2005;162(7):1299-304.

14. Bakker A, Maertens KJ, Van Son MJ, Van Loey NE. Psychological consequences of pediatric burns from a child and family perspective: a review of the empirical literature. Clin Psychol Rev. 2013;33(3):361-71.

15. Bartlett N, Yuan J, Holland AJ, Harvey JG, Martin HC, La Hei ER, et al. Optimal duration of cooling for an acute scald contact burn injury in a porcine model. J Burn Care Res. 2008;29(5):828-34

16. Cuttle L, Kempf M, Kravchuk O, Phillips GE, Mill J, Wang XQ, et al. The optimal temperature of first aid treatment for partial thickness burn injuries. Wound Repair Regen. 2008;16(5):626-34.

17. Cuttle L, Kempf M, Liu PY, Kravchuk O, Kimble RM. The optimal duration and delay of first aid treatment for deep partial thickness burn injuries. Burns. 2010;36(5):673-9.

18. Fein M, Quinn J, Watt K, Nichols T, Kimble R, Cuttle L. Prehospital paediatric burn care: new priorities in paramedic reporting. Emerg Med Australas. 2014;26(6):609-15.

19. Cuttle L, Pearn J, McMillan JR, Kimble RM. A review of first aid treatments for burn injuries. Burns. 2009;35(6):768-75.

20. Yuan J, Wu C, Holland AJ, Harvey JG, Martin HC, La Hei ER, et al. Assessment of cooling on an acute scald burn injury in a porcine model. J Burn Care Res. 2007;28(3):514-20.

21. Wright EH, Harris AL, Furniss D. Cooling of burns: mechanisms and models. Burns. 2015;41(5):882-9.

22. Australian and New Zealand Burn Association (ANZBA). Emergency management of severe burns. 13th ed. Albany Creek: ANZBA; 2009.

23. Jandera V, Hudson DA, de Wet PM, Innes PM, Rode $\mathrm{H}$. Cooling the burn wound: evaluation of different modalities. Burns. 2000;26(3):265-70. 
24. Coats TJ, Edwards C, Newton R, Staun E. The effect of gel burns dressings on skin temperature. Emerg Med J. 2002;19(3):224.

25. Cleland H. Thermal burns: assessment and acute management in the general practice setting. Aust Fam Physician. 2012;41(6):372-5.

26. Flanagan $\mathrm{M}$. Wound healing and skin integrity: principles and practice. Chichester: Wiley-Blackwell; 2013.

27. Goodwin NS, Spinks A, Wasiak J. The efficacy of hydrogel dressings as a first aid measure for burn wound management in the pre-hospital setting: a systematic review of the literature. Int Wound J. 2016;13(4):519-25.

28. Cho YS, Choi YH. Comparison of three cooling methods for burn patients: a randomized clinical trial. Burns. 2017:43(3):502-8.

29. Allison K. The UK pre-hospital management of burn patients: current practice and the need for a standard approach. Burns. 2002;28(2):135-42.

30. Walker A, Baumber R, Robson B. Pre-hospital management of burns by the UK fire service. Emerg Med J. 2005;22(3):205.

31. Cuttle $L$, Kravchuk $O$, Wallis $B$, Kimble RM. An audit of first-aid treatment of pediatric burns patients and their clinical outcome. J Burn Care Res. 2009; 30(6):1028-34

32. Hyland EJ, Harvey JG, Holland AJA. Burn first aid - the weird and wonderful. Poster presented at the Australian and New Zealand Burns Association 37th annual scientific meeting, Fremantle, Western Australia, Australia, 15-18 October 2013.

33. Burd A. Evaluating the use of hydrogel sheet dressings in comprehensive burn wound care. Ostomy Wound Manage. 2007;53(3):52-62.

34. Kandel ER, Schwartz JH, Jessell TM. Principles of neural science. 4th ed. New York: McGraw-Hill Health Professions Division; 2000.

35. Wang Y, Beekman J, Hew J, Jackson S, Issler-Fisher AC, Parungao R, et al. Burn injury: challenges and advances in burn wound healing, infection, pain and scarring. Adv Drug Deliv Rev. 2018;123:3-17.

36. Summer GJ, Dina OA, Levine JD. Enhanced inflammatory hyperalgesia after recovery from burn injury. Burns. 2007;33(8):1021-6.

37. Miller K, Rodger S, Kipping B, Kimble RM. A novel technology approach to pain management in children with burns: a prospective randomized controlled trial. Burns. 2011;37(3):395-405.

38. Kipping B, Rodger S, Miller K, Kimble RM. Virtual reality for acute pain reduction in adolescents undergoing burn wound care: a prospective randomized controlled trial. Burns. 2012;38(5):650-7.

39. Gee Kee E, Kimble RM, Cuttle L, Stockton K. Comparison of three different dressings for partial thickness burns in children: study protocol for a randomised controlled trial. Trials. 2013:14:403.

40. Wiseman J, Simons M, Kimble R, Ware R, McPhail S, Tyack Z. Effectiveness of topical silicone gel and pressure garment therapy for burn scar prevention and management in children: study protocol for a randomised controlled trial. Trials. 2017:18:72.

41. Miró J, Huguet A. Evaluation of reliability, validity, and preference for a pediatric pain intensity scale: the Catalan version of the Faces Pain ScaleRevised. Pain. 2004;111(1):59-64.

42. Stinson NJ, Kavanagh NT, Yamada NJ, Gill NN, Stevens NB. Systematic review of the psychometric properties, interpretability and feasibility of selfreport pain intensity measures for use in clinical trials in children and adolescents. Pain. 2006;125(12):143-57.

43. Finley GA, McGrath PJ. Measurement of pain in infants and children. Seattle: IASP Press; 1998

44. Hicks CL, von Baeyer CL, Spafford PA, van Korlaar I, Goodenough B. The Faces Pain Scale - Revised: toward a common metric in pediatric pain measurement. Pain. 2001;93(2):173-83.

45. Tomlinson D, von Baeyer CL, Stinson JN, Sung L. A systematic review of faces scales for the self-report of pain intensity in children. Pediatrics. 2010; 126(5):e1168-98.

46. Merkel S, Voepel-Lewis T, Malviya S. Pain assessment in infants and young children: the FLACC scale: a behavioral tool to measure pain in young children. Am J Nurs. 2002;102(10):55-8.

47. Babl EF, Crellin PD, Cheng PJ, Sullivan PT, O'Sullivan PR, Hutchinson PA. The use of the faces, legs, activity, cry and consolability scale to assess procedural pain and distress in young children. Pediatr Emerg Care. 2012; 28(12):1281-96.

48. Goodenough B, Champion GD, Laubreaux L, Tabah L, Kampel L. Needle pain severity in children: does the relationship between self-report and observed behaviour vary as a function of age? Aust J Psychol. 1998;50(1):1-9.

49. Goodenough B, Addicoat L, Champion GD, Mclnerney M, Young B, Juniper $K$, et al. Pain in 4- to 6-year-old children receiving intramuscular injections: a comparison of the Faces Pain Scale with other self-report and behavioral measures. Clin J Pain. 1997;13(1):60-73.

50. Crellin D, Sullivan TP, Babl FE, O'Sullivan R, Hutchinson A. Analysis of the validation of existing behavioral pain and distress scales for use in the procedural setting. Paediatr Anaesth. 2007;17(8):720-33.

51. Green $A D$, Linares YRM, Garcia Peña MB, Greenberg LB, Baker LR. Randomized comparison of pain perception during radial head subluxation reduction using supination-flexion or forced pronation. Pediatr Emerg Care. 2006;22(4):235-8.

52. McErlean M, Bartfield JM, Karunakar TA, Whitman MCW, Turley DM. Midazolam syrup as a premedication to reduce the discomfort associated with pediatric intravenous catheter insertion. J Pediatr. 2003;142(4):429-30.

53. Atchison NE, Osgood PF, Carr DB, Szyfelbein SK. Pain during burn dressing change in children: relationship to burn area, depth and analgesic regimens. Pain. 1991:47(1):41-5.

54. Gee Kee EL, Kimble RM, Stockton KA. 3D phtography is a reliable burn wound area assessment tool compared to digital planimetry in very young children. Burns. 2015;41(6):1286-90.

55. Stockton KA, McMillan CM, Storey KJ, David MC, Kimble RM. 3D photography is as accurate as digital planimetry tracing in determining burn wound area. Burns. 2015;41(1):80-4.

56. Van Der Wal M, Bloemen M, Verhaegen $P$, Tuinebreijer $W$, De Vet $H$, Van Zuijlen P, et al. Objective color measurements: clinimetric performance of three devices on normal skin and scar tissue. J Burn Care Res. 2013;34(3):e187-94.

57. Tyack Z, Ziviani J, Kimble R, Plaza A, Jones A, Cuttle L, et al. Measuring the impact of burn scarring on health-related quality of life: development and preliminary content validation of the Brisbane Burn Scar Impact Profile (BBSIP) for children and adults. Burns. 2015;41(7):1405-19.

58. Everett T, Parker K, Fish J, Pehora C, Budd D, Kelly C, et al. The construction and implementation of a novel postburn pruritus scale for infants and children aged five years or less: introducing the Toronto Pediatric Itch Scale. J Burn Care Res. 2015;36(1):44-9.

59. Stevens $K$, Ratcliffe J. Measuring and valuing health benefits for economic evaluation in adolescence: an assessment of the practicality and validity of the Child Health Utility 9D in the Australian adolescent population. Value Health. 2012;15(8):1092-9.

60. Ratcliffe J, Huynh E, Chen G, Stevens K, Swait J, Brazier J, et al. Valuing the Child Health Utility 9D: using profile case best worst scaling methods to develop a new adolescent specific scoring algorithm. Soc Sci Med. 2016;157:48-59.

61. Kirschbaum C, Hellhammer DH. Salivary cortisol in psychobiological research: an overview. Neuropsychobiology. 1989;22(3):150-69.

62. Ginsberg L, Ludman PF, Anderson JV, Burrin JM, Joplin GF. Does stressful venepuncture explain increased midnight serum cortisol concentration? Lancet. 1988:332(8622):1257.

63. Levine A, Zagoory-Sharon O, Feldman R, Lewis JG, Weller A. Measuring cortisol in human psychobiological studies. Physiol Behav. 2007;90(1):43-53.

64. Nater UM, Rohleder N. Salivary a-amylase as a non-invasive biomarker for the sympathetic nervous system: current state of research. Psychoneuroendocrinology. 2009;34(4):486-96.

65. Gee Kee E, Stockton K, Kimble RM, Cuttle L, McPhail SM. Cost-effectiveness of silver dressings for paediatric partial thickness burns: an economic evaluation from a randomized controlled trial. Burns. 2017;43(4):724-32.

66. Brown NJ, David M, Cuttle L, Kimble RM, Rodger S, Higashi H. Costeffectiveness of a nonpharmacological intervention in pediatric burn care. Value Health. 2015;18(5):631-7.

67. Brown NJ, Kimble RM, Gramotnev G, Rodger S, Cuttle L. Predictors of re-epithelialization in pediatric burn. Burns. 2014;40(4):751-8.

68. De Young AC, Kenardy JA, Cobham VE, Kimble R. Prevalence, comorbidity and course of trauma reactions in young burn-injured children. J Child Psychol Psychiatry. 2012;53(1):56-63.

69. Cuttle L, Kimble RM. First aid treatment of burn injuries. Wound Pract Res. 2010;18(1):6-10 2-3.

70. Lonie S, Baker P, Teixeira RP. Healing time and incidence of hypertrophic scarring in paediatric scalds. Burns. 2017;43(3):509-13. 